\title{
Metaplastic Carcinoma with Foci of Chondrosarcoma in Breast: A Rare Case
}

\section{Poorva Vias, Awadhesh Kumar Pandey, Kislay Dimri, Ranjeev Bhagat}

Department of Radiation Oncology, GMCH-32, Chandigarh, India.

\begin{abstract}
Metaplastic carcinoma of breast is a rare form of tumour in which there is a mixture of malignant mesenchymal and epithelial elements. In these tumours cancerous cells of epithelial origin are found in combination with non-specific malignant spindle cells of mesenchymal origin. It is an aggressive form of cancer and usually appears in late stages.
\end{abstract}

Keywords: Metaplasia- Chondrosarcoma- Breast carcinoma

Asian Pac J Cancer Care, 4 (4), 131-133

\section{Introduction}

Metaplastic carcinomas are rare forms of breast carcinomas accounting for less than $0.3 \%$ of invasive breast cancers [1]. Metaplastic carcinoma is a generic term for breast carcinoma of ductal type in which the predominant component of the neoplasm has an appearance other than glandular and epithelial cells.

Metaplastic carcinomas need to be in the differential diagnosis of lesions in the breast. A definitive diagnosis can be reached with core biopsies. Immunohistochemistry can helpful in the diagnosis of metaplastic carcinomas.

Compared to infiltrating duct cell carcinoma, the prognosis of metaplastic carcinoma is poor. The optimal treatment is unknown. Hormonal therapy is usually ineffective and has no role as most of these are hormone receptor negative.

We had a case of metaplastic carcinoma breast with chondrosarcomatous foci at our institute.

Although clinical features of metaplastic carcinomas may mimic breast carcinomas in some ways, therapy and prognosis are different.

\section{Case Report}

A 45 year old female presented with a lump in right breast for about 6 months with history of discharge from nipple. On examination, a fungating mass was present in the right upper outer quadrant of breast with discharge from nipple. The palpable mass was not
Submission Date: 04/18/2019 Acceptance Date: 07/11/2019

fixed to the underlying structures. There was no axillary lymphadenopathy and contralateral breast showed no abnormality. Rest of systemic examination was normal. Diagnosis of carcinoma breast was suspected.

Routine investigations were normal including blood investigations, chest X-Ray, USG abdomen. Bone scan showed no evidence of skeletal metastasis (Figure 1). Biopsy was done outside and a very tiny tissue was reviewed at our institute which showed infiltrating duct cell carcinoma.

PET-CT (Figure 2) showed large FDG-avid soft tissue density mass lesion of size $4.8 \times 3.6 \mathrm{~cm}$ SUV $\max 12.2$ with central necrosis in upper medial quadrant of right breast parenchyma abutting overlying skin. Few mildly FDG avid right axillary lymph node $1.5 \times 0.7 \mathrm{~cm} \mathrm{SUV}$ $\max <2.0$ noted.

Because of locally advanced disease, patient was started on neoadjuvant chemotherapy. There was a partial response to chemotherapy. Patient was taken up for right modified radical mastectomy with axillary lymph node dissection. An ulceroproliferative growth was identified measuring $4.3 \times 3 \times 1.5 \mathrm{~cm}$. On cut section, a tumour is identified corresponding to ulceroproliferative growth on skin measuring $3.2 \times 2.5 \times 1.8 \mathrm{~cm}$.

On microscopic examination (Figure 3 and 4), tumour cells were moderately pleomorphic with vesicular chromatin, inconspicuous nucleoli and moderate amount of cytoplasm. Malignant mesenchymal elements in form of malignant cartilage with high cellularity, hyperchromatic

Corresponding Author:

Dr. Ranjeev Bhagat

Department of Radiation Oncology, GMCH-32, Chandigarh, India.

Email: dr.ranjeevbhagat@gmail.com 


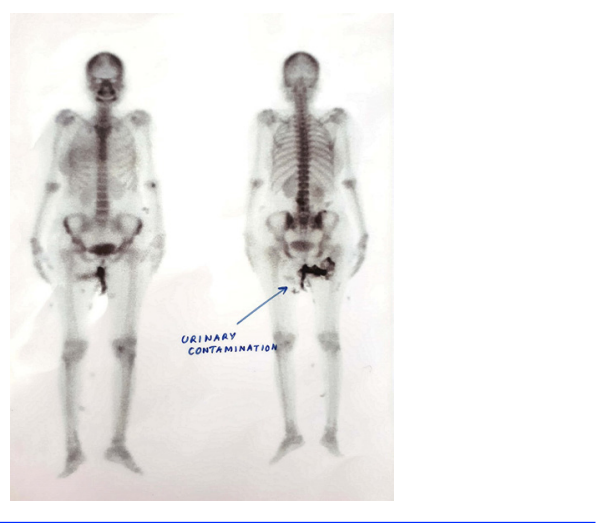

Figure 1. Bone Scan Showing Normal Study

and pleomorphic nuclei and few binucleated chondrocytes were also seen. At places, stroma appeared chondromyxoid, and large chondrosarcomtous areas were seen. Six lymph nodes were isolated and all were free of tumour. Final impression was made of metaplastic carcinoma with focus of chondrosarcoma with lymphovascular emboli. The tumour cells were negative for estrogen and progesterone receptors.

\section{Discussion}

Metaplastic carcinomas of breast are uncommon tumours representing about $0.3 \%$ of invasive carcinomas of breast [1]. These tumours represent a heterogeneous group of malignant tumours in which part or all of the carcinomatous epithelium is transformed into a non-glandular metaplastic growth process [1]. Various types of metaplastic carcinomas have been reported like sarcomatoid carcinoma, spindle cell carcinoma, carcinoma with osteoclast-like giant cells and squamous cell carcinoma. It is important to identify these tumours as they are triple-negative, have an aggressive nature and require a change in the management.

Metaplastic carcinomas includes various categories:

- Sarcomatoid carcinoma, carcinosarcomas and matrix producing carcinomas

- Spindle cell carcinomas

- Carcinomas with osteoclast like giant cells

- Squamous cell carcinoma

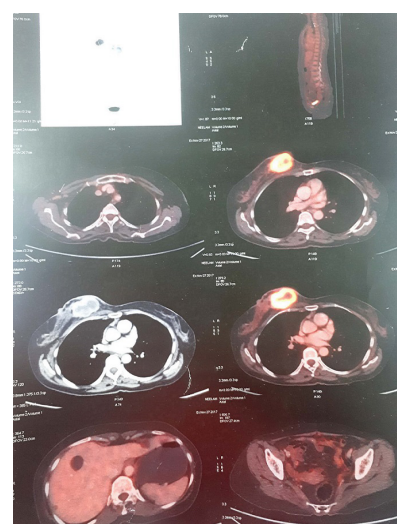

Figure 2. PET-Ct Howing FDG Avidlesion in Left Breast

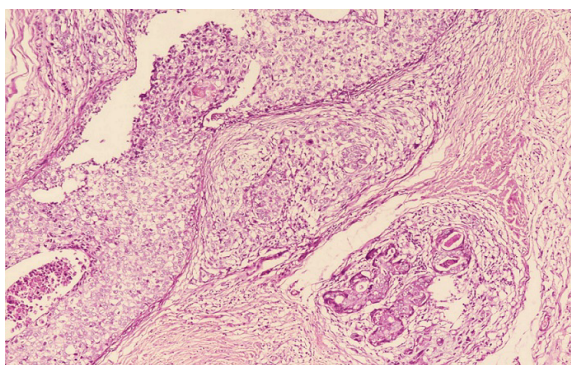

Figure 3. Low Power View of Infiltrating Duct Carcinoma with Insitu Component

- Melanocytic differentiation

- Choriocarcinomatous features

- Pleomorphic carcinomas.

The mean patient age for metaplastic carcinomas of breast is 47.6 years [2]. Grossly, these tumours form large, firm, nodules having a median size of $5 \mathrm{~cm}$. Fixity to skin or deep fascia is not uncommon as seen in our patient. Lymph node involvement is uncommon. Hematogenous spread is commonly explained by sarcomatous element, leading to metastasis to lung, liver, brain and bone.

Primary chondrosarcoma occurring in the breast is extremely rare. It contains chondrosarcomatous areas throughout the tumour and arises from the breast itself rather than from underlying bone or cartilage. Microscopically the tumour shows chondroid areas with cellular atypia and pleomorphism which has to be clearly differentiated from matrix producing metaplastic carcinoma [3] and also from malignant cystosarcoma phyllodes with chondroid differentiation [4].

Differentiation froms metaplastic carcinoma is possible by absence of direct transition between carcinomatous and mesenchymal component in chondrosarcoma. Further the sarcoma like elements in metaplastic carcinoma though acquire vimentin positivity, still retain epithelial markers [5].

Differentiation form malignant cystosarcoma phyllodes with predominant chondrosarcomatoid component can be extremely difficult. Most mammary tumours with areas of chondroid metaplasia show benign histological appearance.

IHC can be helpful in diagnosis, but since cytokeratin expression may be focal, a panel of anti-cytokeratin antibodies should be performed including broad spectrum cytokeratins and basal cytokeratins such as Cytokeratin $5 / 6$, which are most sensitive and specific in metaplastic

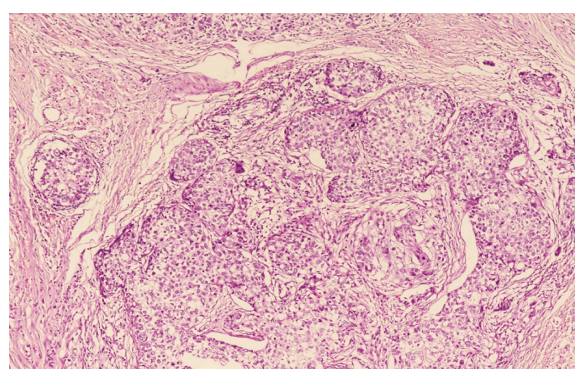

Figure 4. Low Power View of the Malignant Cartilage 
carcinomas [6].

Surgery remains the mainstay of treatment for most sarcomatoid tumors [7]. Multimodality treatment may decrease local and systemic recurrence rates of somatic sarcomas [8].

The tumour is usually negative for any of the hormonal receptors which support the theory that adjuvant therapy with estrogen antagonists and other hormone therapy have no role in treatment of mammary sarcomas.

Bae et al. reviewed 47 metaplastic breast carcinoma and 1346 infiltrating duct carcinomas. They concluded that metaplastic carcinomas are associated with a larger tumour size, lower lymph node involvement, higher histological and nuclear grade, high triple negativity, higher p53, CK 5/6 and EGFR expression, worse prognosis with a disease free survival rate being $78.1 \%$ in metaplastic breast carcinomas compared to $91 \%$ in infiltrating duct carcinomas [9].

In a series of 43 patients with $\mathrm{MBC}$, Dave et al. reported a $10.5 \%$ rate of local recurrence for patients receiving lumpectomy and adjuvant radiation. Total radiation consisted of 50-66 Gy [10].

In conclusion, there is no standard treatment for metaplastic breast cancers due to its rarity and heterogeneity, but histologically specific treatment modalities may offer some benefit. Multimodality treatment is to be used in such cases. Further trials for treatment are needed but are unlikely to accomplish due to its rarity.

\section{References}

1. Ellis IO, Pinder SE, Lee AH. Tumors of the breast. In: Fletcher C, editor. Diagnostic Histopathology of Tumors. 3 rd ed. USA: Elsevier Limited; 2007. p. 938-9

2. Wargotz ES, Norris HJ. Metaplastic carcinoma of the breast I. Matrix producing carcinoma. Hum Pathol 1989;20:628-35.

3. Iihara H, Machinami R, Kubotas S, Itoyama S, Sato A. Malignant cystosarcoma phyllodes tumour of the breast mainly composed of chondrosarcoma - a case report. Gen Diagn Pathol 1997;142:241-5.

4. Vandenhaute B, Validire P, Veilleux C, Voelh P, Zafrani B. Breast carcinoma with chondroid metaplasia. Ann Pathol 1995;15:53-8.

5. Callery CD, Rosen PP, Kinne DW. Sarcoma of the breast. A study of 32 patients with reappraisal of classification and therapy. Ann Surg 1985;201:527-32.

6. Leibl S, Moinfar F. Metaplastic breast carcinomas are negative for Her-2 but frequently express EGFR (Her1): potential relevance to adjuvant treatment with EGFR tyrosine kinase inhibitors? Journal of Clinical Pathology. 2005;58(7):700-704.

7. Rosen PP. Sarcoma. In: Rosen PP, editor. Rosen's Breast Pathology. Philadelphia: Lippincott Williams \& Wilkins; 2001. pp. 863-1.

8. Bae SY, Lee SK, Koo MY, Hur SM, Choi MY, Cho DH, et al. The prognoses of metaplastic breast cancer patients compared to those of triple-negative breast cancer patients. Breast Cancer Res Treat 2011;126:471-8.

9. Rungta S, Kleer CG. Metaplastic carcinomas of the breast: Diagnostic challenges and new translational insights. Arch Pathol Lab Med 2012;136:896-900.

10. Dave G, Cosmatos H, Do T, Lodin K, Varshney D.
Metaplastic carcinoma of the breast: a retrospective review. International Journal of Radiation Oncology Biology Physics. 2006;64(3):771-775.

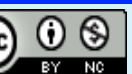

This work is licensed under a Creative Commons AttributionNon Commercial 4.0 International License. 